\section{Coûts de la santé en Suisse}

\author{
F. Herrmann, ${ }^{b, e}$ P. Chastonay, ${ }^{a, c} P$. Chopard ${ }^{b, f}$ E. Chamot, ${ }^{a}$ \\ P. Garnerin, ${ }^{b, g}$ P. Bovier, ${ }^{b, d}$ T. Perneger ${ }^{a, b}$
}

a Institut de médecine sociale et préventive, Université de Genève

b Unité qualité des soins, Hôpitaux Universitaires de Genève

c Unité de développement et de recherche en éducation médicale

d Département de médecine communautaire,

Hôpitaux Universitaires de Genève

e Hôpital de Gériartrie, HUG

f Département de médecine interne, HUG

g Division d'anesthésiologie, HUG

\section{Résumé}

Cet article s'intéresse aux dizaines de milliards de francs consacrés chaque année à la santé en Suisse. Il décrit l'augmentation des coûts de la santé depuis les années soixante et les flux financiers entre les différents acteurs du système de santé. Il termine sur une réflexion concernant la justification des dépenses et une comparaison avec d'autres pays.

Figure 1

Evolution des coûts de la santé en Suisse entre 1970 et 1998, en proportion du PIB [1].

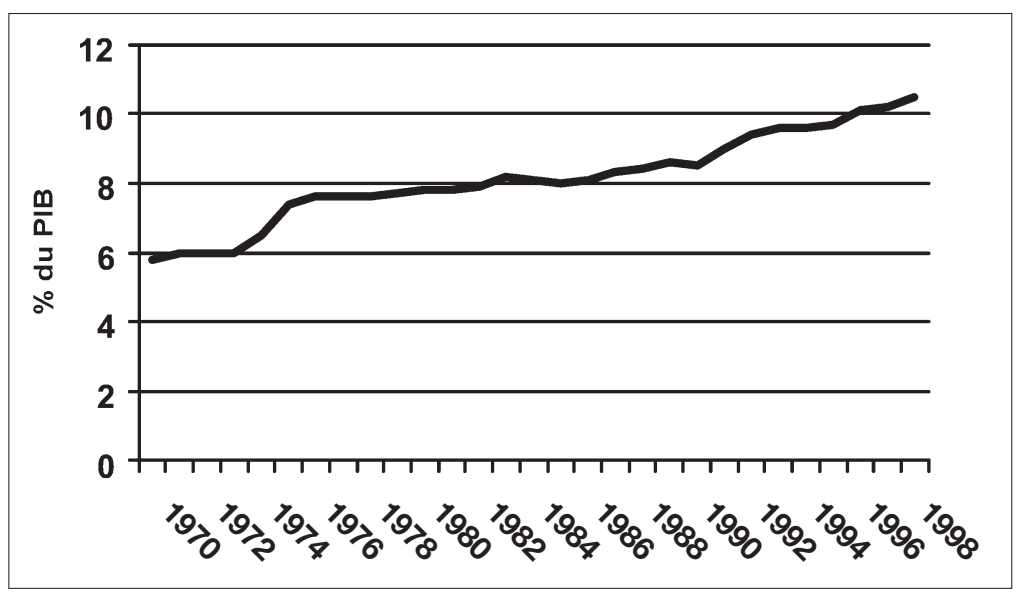

Correspondance:

Dr Philippe Chastonay

Centre médical universitaire

Institut de médecine sociale et préventive

Rue Michel-Servet

CH-1211 Genève 4

\section{Introduction}

La santé coûte trop cher! Maintes fois répétée, cette affirmation est actuellement au centre de débats contradictoires dans notre pays. Mais que veut-on dire par là? Les coûts de la santé augmentent-ils vraiment? Qui supporte le poids financier du système de santé en Suisse? Comment se répartissent les coûts de la santé dans notre pays? En a-t-on pour son argent? Nous allons tenter de donner des éléments de réponse à ces questions.

\section{Augmentation des coûts de la santé}

L'importance du secteur de la santé n'a cessé de croître dans l'économie de notre pays ces dernières années. Si en 1960 les coûts de la santé étaient de 1,9 milliards, ils dépassaient les 10 milliards 15 ans plus tard, et doublaient entre 1985 et 1998 pour atteindre 39,8 milliards [1]. Rapportés au produit intérieur brut (PIB, cf. article "Survol du système suisse de santé»), les coûts de la santé sont passés de 5,7\% du PIB en 1970 à 10,5\% en 1998, soit un doublement en l'espace d'un quart de siècle (fig. 1). Une évolution comparable a été observé dans la plupart des pays industrialisés.

En dehors de la tendance générale à l'augmentation au fil des années, des changements rapides peuvent également survenir. Par exemple, en période de crise économique entre 1973 et 1976 et entre 1990 et 1993, la part des coûts de la santé s'élève rapidement, car le dénominateur (PIB) est directement affecté par la crise et cesse d'augmenter, alors que les dépenses de santé sont relativement incompressibles. A l'opposé, en période de forte croissance, l'augmentation éventuelle des coûts de la santé peut-être contenue par les effets d'une croissance encore plus élevées dans les autres domaines d'activité économique (période des années 80 ).

\section{Flux financiers dans le système de santé}

Lorsqu'on connaît le coût global d'un système de santé, on n'a pas encore dit où va l'argent, qui paye les factures, et qui supporte en fin de compte la charge financière. Ces trois questions sont abordées ici.

Où va l'argent?

Sur les 39,8 milliards dépensés pour la santé en 1998, plus de la moitié l'ont été par les établissements hospitaliers (hôpitaux, maisons pour personnes âgées et établissements pour handicapés) (fig. 2). Les soins ambulatoires (médecins, dentistes, soins à domicile, physiothérapeutes, laboratoires, pharmacie, etc.) représentent globalement 39\%, les médecins étant les bénéficiaires principaux. Les assurances sociales et l'Etat (administration et activités de prévention) engendrent $7 \%$ des dépenses. 


\section{Figure 2}

Coûts du système de santé selon les prestataires de service en 1998, en pour cent [1].

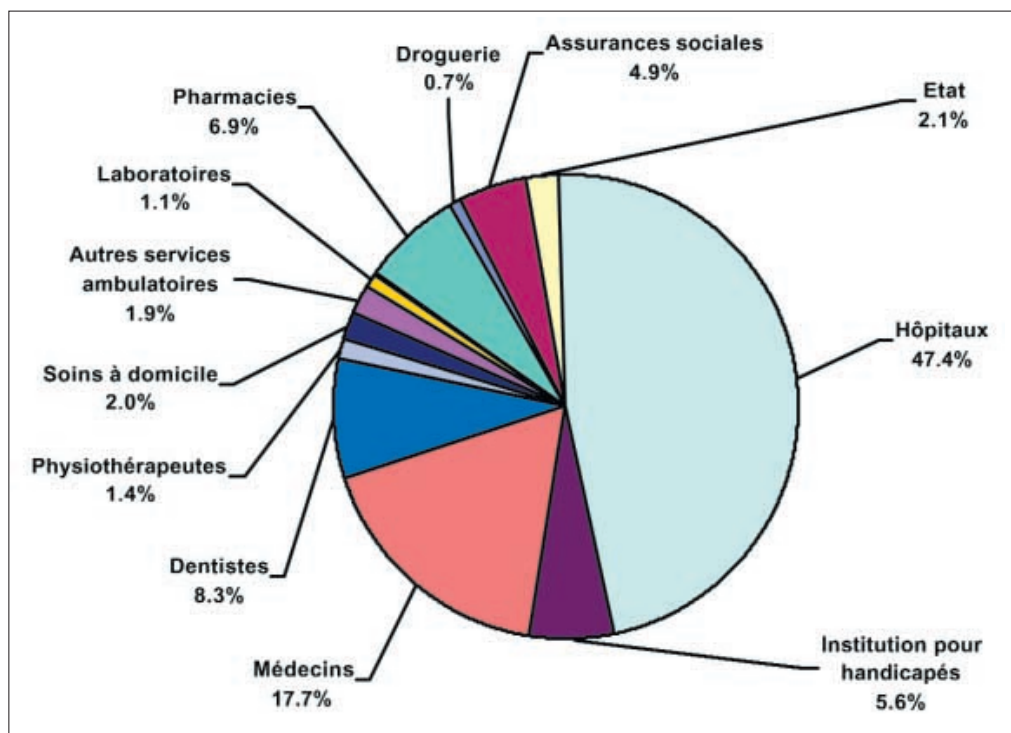

Figure 3

Financement des soins en Suisse selon les agents payeurs en 1998, en pour cent [1].

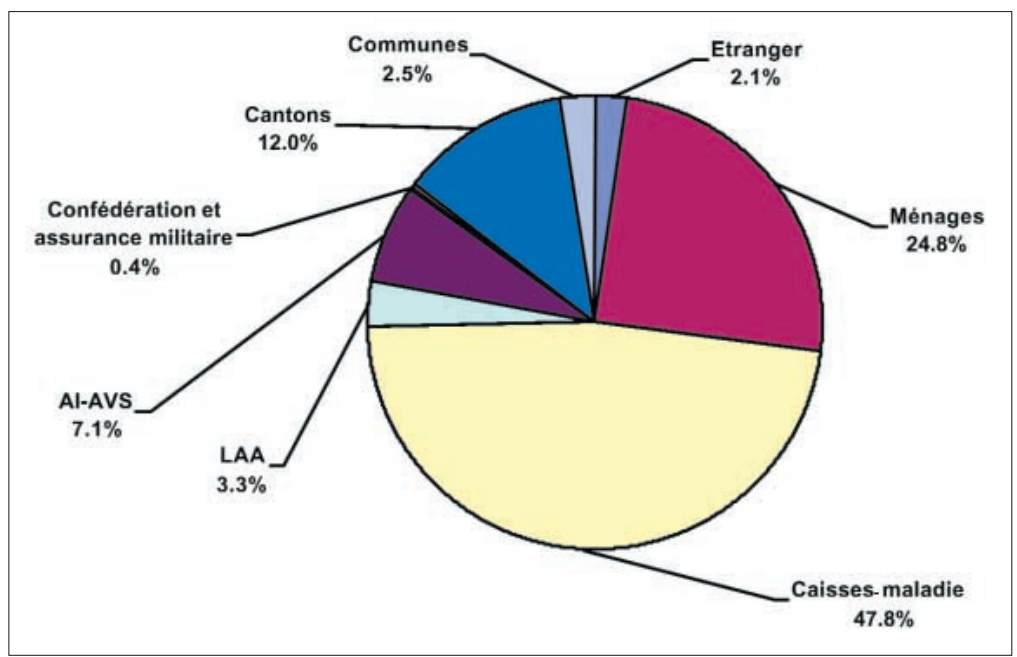

Entre 1960 et 1998, la part attribuable aux établissements hospitaliers est passée de $42 \%$ à 53\%, celle du secteur ambulatoire de $55 \%$ à $40 \%$, et celle de l'administration de $3 \%$ à $7 \%$. Ceci reflète l'hospitalocentrisme croissant des soins, ainsi que le rôle accru de l'état et des assureurs.

Qui paie les factures?

En 1998, les caisses-maladie (assurance de base) couvraient près de la moitié des frais et les ménages payaient directement un quart de ceux-ci (fig. 3). La contribution des ménages comprend leur participation directe aux frais ainsi que le paiement direct pour des prestations non-couvertes par l'assurance de base (assurances complémentaires facultatives, soins dentaires, etc.). L'Etat contribue également au finance- ment, notamment sous forme de subventions aux établissements hospitaliers. Le reste se répartit entre les autres assurances sociales (LAA, AI-AVS) et les personnes domiciliées hors de Suisse.

Entre 1960 et 1998, la part supportée par les assurances sociales (caisses-maladie, LAA, AI-AVS) est passée de $28 \%$ à $58 \%$. Ce progression est liée à l'augmentation de la couverture pour la maladie de la population (en 1960, 28\% de la population suisse n'était pas assurée, actuellement pratiquement 100\% de la population est assurée), à l'élargissement du catalogue de prestations couvertes par la LAMal en 1996, et à la diminution progressive des contributions des pouvoirs publics, dont la part est passée de $23 \%$ à $15 \%$.

De qui vient l'argent?

Celui qui paie la facture n'est pas nécessairement celui qui supporte réellement les coûts. Par exemple, un assureur ne fait que redistribuer les primes provenant des assurés. Prenant ceci en considération, les ménages supportent près des deux tiers des coûts du système de santé en Suisse, et l'Etat un quart (fig. 4). Les entreprises, par leurs contributions sociales, couvrent le reste. Notons qu'on pourrait aller plus loin, et répartir la part de l'Etat, qui est financée par l'impôt, entre les contribuables, à savoir les ménages et les entreprises. Finalement, seul le rendement des intérêts sur les primes versées aux assurances sociales $(1 \%)$ et les frais médicaux des personnes non résidantes en Suisse ne reposent pas sur les citoyens suisses.

Depuis 1960, la part supportée par les ménages a progressé de $62 \%$ à $66 \%$, et celle de l'Etat est passée de $30 \%$ à $24 \%$.

Cette analyse illustre le phénomène de flux financiers entre les différents acteurs; les dépenses de l'un sont les revenus de l'autre. Il faut se souvenir que les prestataires de soins (hôpitaux, médecins, firmes pharmaceutiques, etc.), qui "consomment" les ressources financières en bout de chaîne, recyclent leurs revenus dans le système économique, notamment en payant leurs employés et leurs fournisseurs, précisément ceux qui supportent les coûts. Ainsi la boucle est bouclée.

\section{Les coûts de la santé en Suisse sont-ils justifiés?}

Les dépenses de santé en Suisse sont-elles excessives, adéquates, ou insuffisantes? La réponse à cette question est plus politique qu'économique. Nous nous contenterons de donner quelques éléments de discussion selon le point de vue des économistes.

Le point de départ de la réflexion économique est que les ressources à disposition d'un pays sont en quantité limité. Ainsi, une augmentation de l'utilisation des ressources dans un secteur (par exemple la santé) ne pourra se faire qu'au détriment des autres, tels que l'éducation, les transports, les loisirs, etc. Lorsque l'Etat décide de construire ou rénover un hôpital, il devra renoncer à un nouveau tronçon d'au- 


\section{Figure 4}

Financement selon l'agent supportant la charge économique en 1998, en pour cent [1].

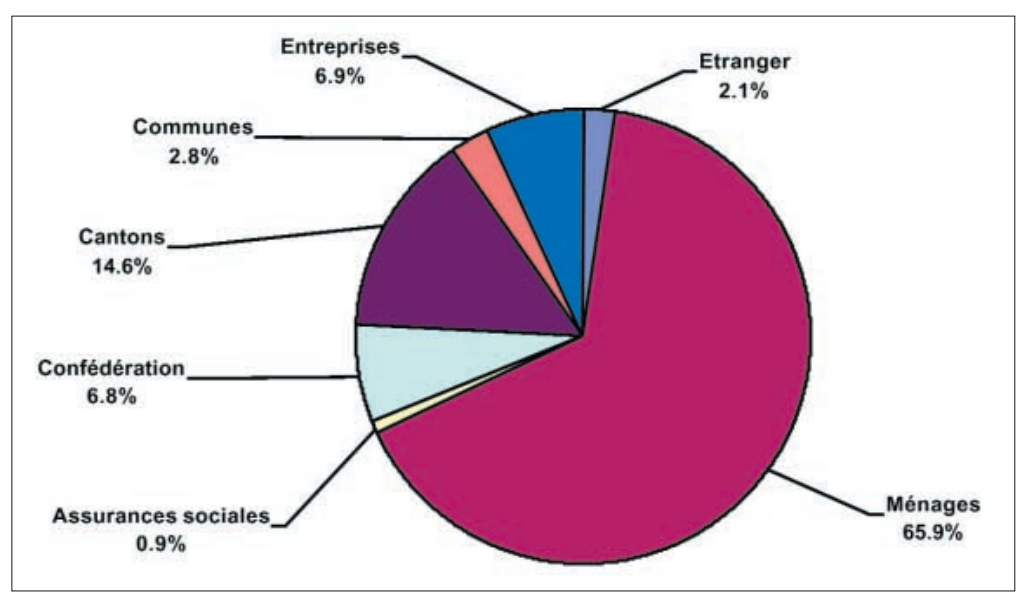

\section{Figure 5}

Relation entre espérance de vie et dépense de santé par habitant dans le monde en 1990 [2].

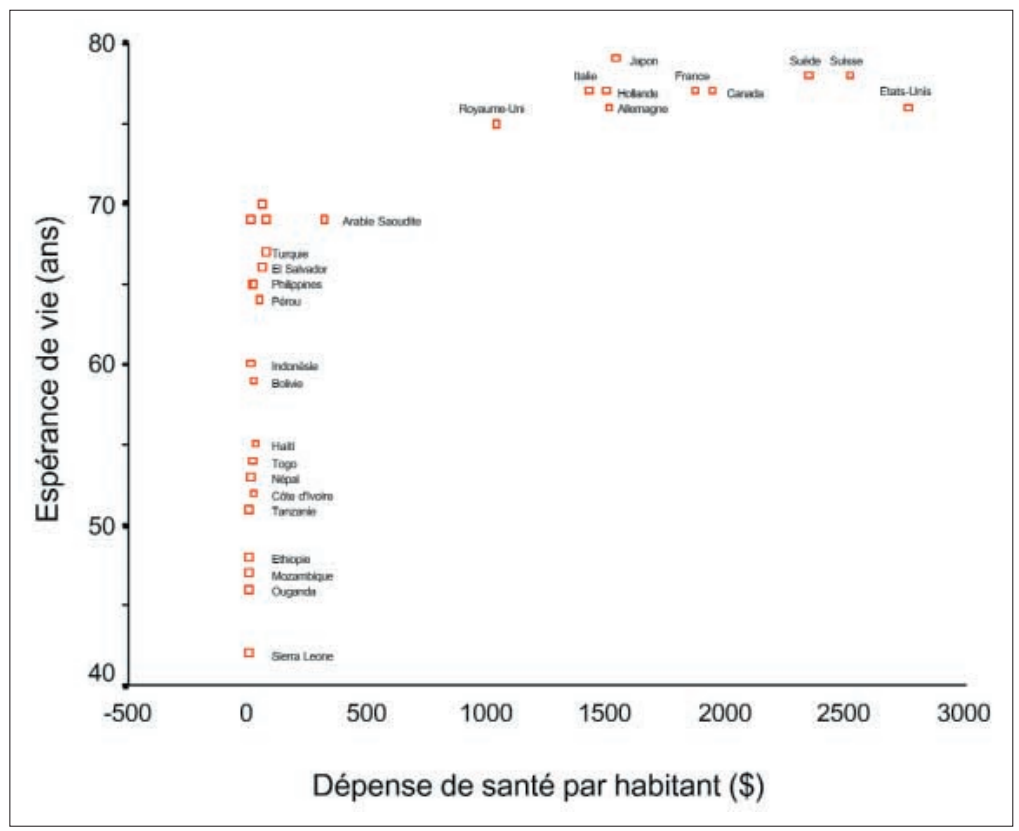

toroute, ou à un autre investissement public. C'est ce qu'on appelle le coût de renonciation ou «opportunity cost", une notion essentielle en économie. Le coût de renonciation ne se chiffre pas en francs, mais représente la valeur de la meilleure alternative à laquelle on a renoncé. Pour un individu qui hésite entre partir en voyage autour du monde et acheter une voiture, et qui finit par choisir le voyage, le coût de renonciation sera la privation de la voiture.

Les choix d'allocation des ressources reflètent donc naturellement les jugements de valeur des individus, et on pourrait dire que les gens pratiquent l'analyse économique comme M. Jourdain fait de la prose. Pour les choix de société, les choses sont plus compliquées, parce que la prise de décision est habituellement négociée, et les arguments des uns et des autres doivent dès lors être explicites. En ce qui concerne les coûts de la santé, on pourrait dire que ceux-ci sont excessifs à partir du moment où la majorité des individus qui bénéficient du système de santé et en supportent la charge estiment qu'un investissement dans d'autres secteurs leur apporterait de plus grands bénéfices.

Le problème est que la mesure des «bénéfices» du système de santé est difficile. Prenons l'espérance de vie. Si on examine la relation entre les dépenses de santé et l'espérance de vie dans différents pays (fig. 5), on constate que les différents pays se répartissent en deux groupes. Dans les pays en voie de développement, les dépenses de santé sont généralement inférieures à US\$ 150 par habitant et par année, et les différences d'espérance de vie sont importantes. Ces différences ne s'expliquent pas entièrement par les dépenses. Ceci suggère que d'autres facteurs que les dépenses de santé influencent l'espérance de vie, comme l'environnement, l'éducation, l'accès à l'eau propre, la sécurité routière, ou l'hygiène alimentaire, qui ne figurent pas sous la rubrique des «dépenses de santé». De plus, on pourrait suspecter que c'est justement parce que les gens meurent jeunes dans certains de ces pays que les dépenses de santé restent relativement modestes, ce qui renverse l'argument de causalité.

En revanche, dans les pays industrialisés, les dépenses en matière de santé sont considérablement plus élevées, mais sans relation apparente avec l'espérance de vie. Par exemple, au Japon et aux Etats-Unis, l'espérance de vie est comparable, mais les dépenses de santé varient du simple au double! A noter que la Suisse est en deuxième position, en termes de coûts. Peut-on alors conclure qu'on dépense trop pour la santé dans notre pays? Pas vraiment.

Si l'investissement dans la santé influence peu l'espérance de vie au-delà d'un certain seuil, ceci ne veut pas dire que ces investissements sont inutiles. D'autres indicateurs que la survie, tels que la qualité de vie de la population, pourraient mieux capter les bénéfices obtenus. De plus, le processus des soins présente en lui-même un intérêt pour les patients, quel que soit le résultat final en termes de santé, comme le veut la devise "guérir parfois, soulager souvent, soigner toujours".

La figure 5 illustre par ailleurs une autre notion fondamentale en économie: la loi des retours décroissants. Cette loi dit qu'à mesure qu'on investit davantage dans une activité, les bénéfices obtenus par franc supplémentaire diminuent. Cette "loi», décrite au $18^{\mathrm{e}}$ siècle [3], n'est pas le fruit d'une démonstration théorique, mais résulte d'observations effectuées par les premiers économistes. Cette observation a été confirmée à de nombreuses reprises depuis. 


\section{Conclusion}

Les coûts de la santé en Suisse ont régulièrement augmenté depuis 40 ans, pour atteindre 39,8 milliards de francs en 1998, soit 10,5\% du PIB. Les établissements hospitaliers et autres institutions représentent près de la moitié de ces dépenses et le secteur ambulatoire près de $40 \%$. Les frais sont couverts à raison de $60 \%$ par les assurances sociales (caisses-maladie, LAA, AI-AVS), d'un quart par les ménages et d'environ $15 \%$ par les pouvoirs publics. Toutefois la grande majorité de la charge économique repose en fin de compte sur les ménages, soit par l'entremise des impôts, des cotisations d'assurance, ou des paiements directs.

Si la Suisse possède un système de santé performant, celui-ci est parmi les plus coûteux dans le monde. Savoir si ces dépenses sont justifiées relève d'un choix de société.

\section{Références}

1 Office fédéral de la statistique. Coûts du système de santé. Neuchâtel: OFS; 2000; www.statistique.admin.ch/stat_ch/ ber14/gewe/cout_sante/fkos1002.htm.

2 The World Bank. World Development Report. New York: Oxford University Press; 1993.

3 Baumol WJ, Blinder AS. Microeconomics: principle and policy. Fort Worth, TX: The Dryden Press; 1997. 